\title{
e-Phaïstos
}

e-Phaïstos

Revue d'histoire des techniques / Journal of the history

of technology

II-2 | 2013

Les sources de l'histoire des techniques (2)

\section{Le patrimoine de l'industrie en Inde}

Un débat à entretenir

Industrial Heritage in India : a Much Needed Discussion

Manisha lyer

\section{(2) OpenEdition}

Journals

Édition électronique

URL : http://journals.openedition.org/ephaistos/7265

DOI : 10.4000/ephaistos.7265

ISSN : 2552-0741

Éditeur

IHMC - Institut d'histoire moderne et contemporaine (UMR 8066)

Édition imprimée

Date de publication : 15 décembre 2013

Pagination : 116-123

ISSN : 2262-7340

Référence électronique

Manisha Iyer, « Le patrimoine de l'industrie en Inde », e-Phaïstos [En ligne], II-2 | 2013, mis en ligne le 12 décembre 2019, consulté le 14 décembre 2019. URL : http://journals.openedition.org/ephaistos/7265 ; DOI : 10.4000/ephaistos.7265

Tous droits réservés 


\title{
Le patrimoine de l'industrie en Inde : un débat à entretenir
}

\author{
Manisha Iyer \\ Doctorante - Équipe d'Histoire des Tech- \\ niques / IHMC \\ CNRS - Université de Paris 1 Panthéon- \\ Sorbonne
}

L'Inde reste terra incognita en ce qui concerne l'anthropologie des techniques ou le patrimoine industriel. Le peu d'écrits que nous trouvons sur ces sujets sont dus à des chercheurs étrangers, le pionnier étant Jean Filliozat qui a beaucoup écrit sur la médecine indienne. Grâce aux initiatives du TICCIH et du mAAN (le réseau d'architecture de l'Asie moderne), le patrimoine de l'industrie commence à devenir un sujet d'intérêt pour des jeunes chercheurs professionnels. Jusqu'à présent, la notion de patrimoine industriel est un champ d'étude quasiment inconnu en Inde. Cette discipline ne figure pas dans les programmes proposés par les universités indiennes. De plus, bien qu'il existe de nombreux musées en Inde, aucun ne traite ce sujet. Pourtant, la civilisation indienne est une des plus anciennes civilisations du monde et les fouilles archéologiques montrent des industries anciennes telles que la poterie, ou la métallurgie, depuis la civilisation de la vallée de l'Indus (5000 av. J.-C. - 1900 av. J.-C.). Cependant, avec l'arrivée des pouvoirs européens au cours du XVe siècle, l'industrie en Inde a connu un développement important.

L'Inde a été pendant longtemps un territoire divisé en de nombreux petits royaumes. Alexandre le Grand fut le premier européen à y arriver en 327 av. J-C. L'empire Maurya (322 av. J-C - 180 av. J-C) a été fondé suite à la retraite d'Alexandre le Grand dans l'ouest du sous-continent indien. Afin de faire face aux éventuelles invasions de l'Occident, il a fallu mettre en place un État unifié, ainsi les Maurya régnant sur une grande partie du sous-continent formaient le plus grand royaume de l'Inde. Même si vers 320 av. J-C l'Empire Maurya a réussi à vaincre l'armée grecque laissée par Alexandre, les relations commerciales avec la Grèce ont continué ${ }^{1}$. Le Périple de la mer Erythrée, par exemple, rédigé dans la première moitié du $1^{\mathrm{er}}$ siècle avant Jésus-Christ détaille les relations commerciales entre ces deux pays avec une description élaborée des différents ports maritimes de l'Inde ainsi que les différentes marchandises ${ }^{2}$. Les objets d'art en terre cuite, ivoire et métal étaient les plus échangés. Les fouilles archéologiques effectuées dans plusieurs régions indiennes affirment l'existence d'une production artisanale qui a connu un important succès commercial3.

Il a fallu attendre la découverte de la route maritime par Vasco de Gama en 1498 qui a ouvert les portes du sous-continent indien aux autres puissances européennes, notamment le Royaume-Uni, la France, le Portugal, la Hollande et le Danemark. Cette rencontre joue un rôle très important dans l'histoire politique, sociale, culturelle et industrielle de l'Inde. Bien que la culture indienne soit très influencée par ces différentes cultures occidentales de l'architecture à la gastronomie - vers le milieu du XIX ${ }^{\mathrm{e}}$ siècle, le Royaume-Uni a réussi à prendre contrôle de la plupart du sous-continent indien. Ainsi, l'Inde britannique est devenue la plus peu- 
plée et la plus précieuse des provinces de l'Empire britannique. Jean Filliozat n'a pas tort lorsqu'il dit que l'Inde " est restée sensible au légitime prestige de la science et des techniques modernes, surtout telles qu'elles sont diffusées en anglais ». Tout en gardant les techniques indigènes, l'Inde est parvenue à incorporer la technologie apportée par les «blancs », ce qui a contribué au progrès industriel du pays.

\section{Le patrimoine industriel : une notion oc- cidentale}

Le domaine du patrimoine industriel se développe en tant que dérivation de l'archéologie industrielle dans les années 1950 en Grande Bretagne qui a été le premier pays à vivre la révolution industrielle. L'archéologie industrielle, terme introduit au XVIII siècle par les britanniques, a été créée dans le souci d'inventorier et dans certains cas, de conserver les "monuments" industriels. La première révolution industrielle a laissé des preuves tangibles des ateliers abandonnés, des usines désertées ou des canaux désaffectés. Leur étude constitue alors un nouveau champ de recherche patrimonial. Les "restes industriels" englobent tout ce qui est relatif à l'industrie, matériel et immatériel : les bâtiments, les machines, les outils, les paysages et le savoirfaire. Comme le résume Maurice Daumas, l'archéologie industrielle vise à étudier ce qui n'est pas détruit, mais est abandonné ou délaissé afin de pouvoir déterminer son historicité, apprécier sa valeur et son intérêt et enfin établir un projet de conservation ou de mise-en-valeur4.

Cela fait de l'archéologie industrielle un champ pluridisciplinaire, permettant aux archéologues, historiens, sociologues, et géographes de travailler les uns avec des autres. L'archéologie industrielle est un outil qui aide à mieux comprendre le passé et le présent industriel. Si la notion d'archéologie industrielle a débuté en Grande-Bretagne, les autres pays européens et l'Amérique du Nord, notamment les États-Unis d'Amérique, n'ont pas tardé à s'en saisir. Tandis qu'aux États-Unis, le concept s'est développé au cours des années 1960 à la fin de la société fordiste, la destruction des villes européennes lors de la Seconde Guerre mondiale et l'ampleur du travail nécessaire dans la Reconstruction a suscité la naissance du patrimoine industriel en Grande-Bretagne. En 1973, suite au premier colloque international pour la conservation du patrimoine industriel qui a eu lieu à Ironbridge en Grande-Bretagne, est né le comité international pour la conservation du patrimoine industriel connu sous le nom de TICCIH - une organisation mondiale dont l'objectif est la promotion de la recherche sur le patrimoine industriel et sa protection. Ce n'est que vers la fin des années 1980 que le terme de «patrimoine industriel » finit par devenir une expression globalement acceptée. 5

Du fait que le développement de l'archéologie industrielle se passe au même moment que la décolonisation, la période privilégiée pour l'étude du patrimoine industriel en Occident est généralement acceptée comme la période de la révolution industrielle ${ }^{6}$. Toutefois, nous trouvons souvent des exemples aussi magnifiques et intéressants datant de la période de la colonisation et notamment pour l'Inde où ils correspondent à la période la plus forte de l'expansion britannique. Plusieurs anciennes colonies telles que l'Inde ont ainsi connu une période d'essor entre les années 60 et 80 alors qu'ils cherchaient à avancer et progresser en mettant l'accent sur le développement d'une industrie moderne s'alignant sur les projets nationaux.

\section{Le contexte indien}

Le TICCIH donne la définition suivante : «Le patrimoine industriel comprend les vestiges de la culture industrielle qui sont de valeur historique, sociale, architecturale ou scientifique. Ces vestiges englobent: des bâtiments et des machines, des ateliers, des moulins et des usines, des mines et 
des sites de traitement et de raffinage, des entrepôts et des magasins, des centres de production, de transmission et d'utilisation de l'énergie, des structures et infrastructures de transport aussi bien que des lieux utilisés pour des activités sociales en rapport avec l'industrie (habitations, lieux de culte ou d'éducation). » ${ }^{7}$

En repérant des exemples qui correspondent à cette définition, nous observons que le développement industriel en Inde s'est produit dans trois périodes: de l'Antiquité jusqu'à la colonisation (XVIe siècle); la période moderne qui commence avec la colonisation (du XVIe siècle à 1947) ; après l'Indépendance (à partir de 1947). Il est évident que chaque période a des problèmes d'identification et de valorisation du patrimoine de l'industrie.

\section{La période de l'Antiquité (jusqu'au XVIe siècle)}

Les toutes premières forges de l'Inde du Sud remontent à 1000 av. J.-C. dans les États de Karnataka et Tamilnadu. Les analyses techniques des objets retrouvés dans ces sites montrent que les forgerons étaient capables de fabriquer de grands objets et qu'ils expérimentaient depuis des siècles. Selon le Service d'archéologie de l'Inde, l'extraction de fer existe en Inde depuis le XVIe siècle av. J-C. et vers la première décennie du XII ${ }^{e}$ siècle, cette activité a été pratiquée à une grande échelle en Inde 8 .

\section{La période moderne (du XVIe siècle à 1947)}

En tant que «joyaux de la couronne ${ }^{9}$ de l'empire britannique, l'Inde a été directement influencée par la révolution industrielle de la Grande Bretagne et de l'Europe en générale. Ainsi, une grande partie du développement industriel du pays a lieu à partir de la deuxième moitié du XIXe siècle. C'est aussi l'époque de l'industrie ferroviaire et ceci a contribué largement au développement des villes industrielles indiennes. La plupart des exemples qui sont proposés dans cet article rentrent dans cette période. Bien que l'Inde doive une grande partie de sa progression technique au Royaume Uni, les autres colonisateurs européens y ont également contribué.

\section{Après l'Indépendance (à partir de 1947)}

Après l'Indépendance en 1947, l'Inde continue à se développer et devient petit à petit une puissance économique à partir de la dernière décennie du $\mathrm{XX}^{\mathrm{e}}$ siècle. Pendant cette époque, plusieurs compagnies multinationales s'implantent en Inde. L'économie de l'Inde voit un accroissement important à partir de cette période et continue à se développer à une grande vitesse. Pour nommer quelques compagnies, l'Enfield ou l'Union Carbide constituent de bons exemples de patrimoine industriel de cette période.

\section{Un patrimoine en péril: des exemples}

Pour illustrer cette rencontre qui a largement contribué au développement technique, technologique et économique indien, on a choisi quatre exemples significatifs qui permettent également de comprendre la place actuellement accordée au patrimoine industriel en Inde.

\section{Les filatures de coton de Mumbai (Bombay)}

Le Nord-Ouest de l'Inde est connu pour sa longue tradition de production textile en coton. Pourtant, pendant la colonisation britannique, l'Inde a été obligée d'arrêter toute production de textile coton. Selon les normes commerciales imposées par la Compagnie anglaise des Indes orientales, l'Inde n'avait le droit que d'exporter le coton en Grande-Bretagne pour ensuite importer les textiles fabriqués dans le Lancashire (le comté de Lancaster). Vers la deuxième moitié du XIX ${ }^{\mathrm{e}}$ siècle, sous l'impulsion des entrepreneurs indiens, une industrie de coton émerge. Grâce au soutien des fabri- 
cants britanniques des machines de textiles, ils réussissent à mettre à l'écart la résistance des adversaires britanniques. Ainsi, en 1854, la toute première manufacture de coton - The Bombay Spinning and Weaving Company - est installée. L'Inde a connu un essor dans l'exportation du coton lors de la guerre civile américaine et vers la fin du XIX siècle, il y avait 70 filatures de coton à Mumbaï (ou Bombay). Petit à petit le nombre des usines de textiles augmente et transforme la ville de Mumbaï, qui, de ville portuaire, devient une métropole industrielle. Le quartier où se trouvaient ces usines a été connu sous le nom de Girangaon, signifiant le "village des usines" dans la langue régionale. Vers 1915 , 83 filatures s'y implantent ${ }^{10}$. Les hommes et les femmes travaillaient dans ces implantations. Les patrons de ces usines ont mis à disposition des logements destinés aux ouvriers et à leurs familles. Même s'ils pratiquaient différentes religions, ils participaient à une vie commune. Le quartier de Girangaon est vite devenu une cité ouvrière ou company town. Pourtant, ce phénomène n'a pas duré longtemps : en 1953, le nombre d'usines n'est plus que de $53^{11}$. Auparavant appelé le « Manchester de l'Orient ${ }^{12}$, le paysage de la ville de Mumbaï a été complètement transformé en 1982, suite à la grève de 18 mois pour une augmentation de salaires et primes qui a provoqué la fermeture de la plupart de ces usines ${ }^{13}$. Aujourd'hui, certaines de ces usines sont soit entièrement détruites soit converties en centres commerciaux ou boîtes de nuit ${ }^{14}$.

\section{Les forges de Kumaon \& Burwah}

En 1860, trois suèdois - Carl Gustaf Wittenstrom, Julius Ramsay et Nils Wilhelm Mitander arrivent en Inde pour participer à un projet britannique : établir deux usines sidérurgiques avec la technique suédoise du haut-fourneau. Les deux endroits choisis étaient le village de Decahuri dans les montagnes de Kumaon et le village de Burwah dans la vallée de Narmada. D’après les divisions administratives actuelles de l'Inde, les forges de
Kumaon se situent dans l'État d'Uttaranchal dans le nord de l'Inde ; et les forges de Burwah dans l'État de Madhya Pradesh en Inde centrale.

Bien avant ce projet, ces deux régions avaient une longue histoire dans l'extraction de fer, raison principale de leur sélection. Tandis qu'en Inde, on utilisait le bas-fourneau, la fabrication de fonte se faisait avec les haut-fourneaux en Europe. Cette méthode très répandue en Europe a donc été introduite en Inde par ces trois suédois. Dans la première moitié du XIX ${ }^{\mathrm{e}}$ siècle, de nombreux recensements effectués par les colonisateurs dans les régions de Kumaon et Burwah ont révélé d'importantes ressources naturelles, notamment du charbon et du fer. L'intérêt commercial commun entre les colonisateurs européens, particulièrement le Royaume-Uni, dont l'intérêt principal était l'accélération de l'industrie britannique en Inde, a largement facilité ce projet. Une tâche fascinante mais certainement pas facile. Après beaucoup de problèmes techniques, logistiques, sociaux et culturels, deux usines innovantes ont été établies développant des techniques pionnières dans le contexte indien ${ }^{15}$. Malheureusement, malgré son excellente conception et la plus moderne technologie européenne de l'époque, le projet n'a pas réussi. Les coûts de production étaient disproportionnés et toute activité a dû être arrêtée. Avant même de commencer la pleine production, les usines ont fermé et les suédois ont dû rentrer en Suède. Aujourd'hui, les vestiges de ce projet innovant sont délaissés dans les deux villages. À Kumaon, la structure physique a été complètement détruite et seules des traces au sol sont visibles alors qu'à Burwai, la cheminée et la structure centrale sont en ruines.

\section{Darjeeling Himalayan Railways (DHR)}

La toute première compagnie des chemins de fer à être créée en Inde est l'East Indian Railway (EIR). Fondée en 1845, la compagnie ouvre la première route pour les passagers en 1854 . La ville de Darjeeling est située dans les contreforts de 
l'Himalaya et prend son nom du tibétain Dorje Ling signifiant la « cité de la foudre ». Inauguré en 1881, le Darjeeling Himalayan Railway (DHR) relie la petite ville de New Jalpaiguri à Darjeeling dans l'État de West Bengal, situé dans l'Est de l'Inde. La ville de Darjeeling était la ville estivale des britanniques et c'est cette appréciation de la ville par les britanniques qui a poussé à la construction du plus bel exemple de chemin de fer de montagne pour passagers ${ }^{16}$. Reconnu comme le premier chemin de fer de montagne pour passagers, il a été construit entre 1879 et 1881. Aussitôt surnommé le «Toy Train » ou le «train jouet », le DHR est un chemin de fer à voie étroite, l'écartement des rails étant 610 $\mathrm{mm}$. Le DHR couvre une distance de $88 \mathrm{~km}$ à des altitudes variant de $100 \mathrm{~m}$ à $2200 \mathrm{~m}$. Afin de contourner les problèmes liés à un terrain montagneux d'une beauté exceptionnelle, des solutions «ingénieuses et audacieuses » ont été employées ${ }^{17}$. Étant un chemin de fer de montagne, le plus grand défi de construction était la pente. Au lieu de construire des tunnels, il a été décidé de construire un chemin de fer avec des boucles et des zigzags. Avec 4 boucles et 6 zigzags, le DHR a réussi à surmonter les problèmes de pente. En décembre 1999, l'UNESCO a classé le DHR au patrimoine mondial avec deux autres liaisons ferroviaires en tant que "Chemins de fer de montagne en Inde » pour les raisons suivantes :

" An outstanding example of the influence of an innovative transportation system on social \& economic development of a multicultural region, which was to serve as a model for similar developments in many parts of the world.( C(ii) )

The development of railways in the 19th century had a profound influence on social and economic developments in many parts of the world. This process is illustrated in an exceptional and seminal fashion by the DHR. (C(iv) ) $»^{18}$.
Cette voie ferroviaire est toujours en fonction avec très peu de modifications de ses caractéristiques originales. Aujourd'hui, le DHR est une attraction touristique autant qu'il l'était lors de son ouverture voici 132 ans. C'est probablement pourquoi il est resté préservé pendant toutes ces années. Les touristes indiens tout comme les touristes étrangers visitant la ville de Darjeeling ne manquent guère l'occasion de prendre ce train.

\section{L'usine Royal Enfield ${ }^{19}$}

L'usine de moto Enfield dans la ville de Chennai (auparavant Madras) dans le sud de l'Inde est le meilleur exemple du patrimoine industriel de la période post-moderne. En 1909, la compagnie Royal Enfield crée une révolution dans le monde des motos en fabricant la première moto avec moteur en V. Elle devient aussitôt un nom populaire. Même si depuis 1949 l'Inde importait des motos Royal Enfield, en 1955 l'État indien a passé une grosse commande de 800 motos destinées à sa gendarmerie. Afin de répondre à cette commande énorme pour l'époque, le Enfield India a été créé en 1956 dans la ville de Chennai. Petit à petit, ces motos ont réussi à dominer le marché indien, notamment le marché rural, et sont restées, jusqu'à très récemment, quasiment les seules motos disponibles sur le marché indien. En 1970, la compagnie britannique, Royal Enfield, ferme ses portes et arrête toute production, elle sera dissoute en 1971. Mais l'Inde a racheté la licence d'exploitation et continue jusqu'à présent à fabriquer ces machines. Afin de satisfaire des clients indiens certes, mais aussi internationaux, la compagnie ne cesse pas d'innover et aujourd'hui, cette machine fait partie du patrimoine industriel et culturel de l'Inde. Pour célébrer ce patrimoine industriel, les motoristes enthousiastes - étrangers et indiens - organisent tous les ans, un circuit routier du sud de l'Inde jusqu'à l'Himalaya. 


\section{Conclusion}

Certes, les exemples cités ci-dessus ne constituent pas une liste exhaustive du patrimoine industriel indien, mais sont des témoins de sa richesse. Ils permettent de découvrir les différents paramètres de leur état actuel. Il faut commencer par reconnaître le potentiel du patrimoine industriel en Inde. Des circuits touristiques focalisés sur le patrimoine industriel peuvent contribuer au développement économique. Pour la première fois, en 2011, grâce aux initiatives du TICCIH et du mAAN, un colloque international a été organisé autour des questions du patrimoine de l'industrie. Ce colloque a porté une attention particulière à l'usine de l'Union Carbide India Limited (UCIL), la filiale indienne d'un des plus grands groupes chimiques américains, l'Union Carbide Corporation, située dans la ville de Bhopal. Construite en 1978, l'UCIL, a été bien accueillie, mais a mal tourné. La nuit du 3 décembre 1984 change tout pour la ville et ses habitants lorsque, suite à une explosion, la plus grande catastrophe industrielle du monde s'est produite ${ }^{20}$. Suite à cet accident, l'usine a dû être fermée et aujourd'hui elle est en ruine. La conservation d'un site tel que celui-ci soulève certes des débats polémiques ; cependant, il faut reconnaître que ce site est un exemple unique qui permet de comprendre comment un site à l'histoire troublante et perturbante contribue au patrimoine culturel et industriel d'un pays.

L'objectif de toute conservation historique est de préserver et sauvegarder les vestiges historiques d'un pays afin de pouvoir reconstruire son passé et en ce qui concerne l'histoire industrielle, le progrès technologique qu'il a fait au cours des siècles. Comme nous pouvons le constater, à l'exception du DHR, aucun site n'est identifié comme patrimoine industriel en Inde malgré leur contribution au développement culturel, social et technologique du pays. À ce jour, 29 sites indiens sont classés par l'UNESCO comme patrimoine mondial dont 6 sites sont classés patrimoine naturel et 23 sites patri- moine culturel ${ }^{21}$. À ce jour, il n'existe aucun inventaire du patrimoine industriel. Réaliser un tel inventaire devrait être une priorité. Et pour ceci, il faudra développer les compétences. Le patrimoine industriel pourra être introduit comme une discipline dans les universités indiennes. Avec un potentiel énorme, les architectes, les archéologues, les historiens ou les environnementalistes pourront s'y intéresser.

Des organisations publiques comme l'Archaeological Survey of India (l'organisation pour les études archéologiques de l'Inde) ainsi que des organismes privés tels que l'INTACH (l'organisation nationale indienne pour l'art et le patrimoine culturel) travaillant sur la protection et conservation des sites patrimoniaux ne traitent pas les questions du patrimoine de l'industrie. Seuls les sites qui sont classés "monuments/sites protégés" selon le Monuments and Antiquities Act, 1961, bénéficient d'un système de conservation. En l'occurrence, beaucoup de sites de valeur patrimoniale que ce soit culturelle, architecturale ou industrielle des villes indiennes sont en péril. Du fait que les filatures de Mumbaï ne sont pas classées comme patrimoine, certains bâtiments ont été détruits pour construire des appartements résidentiels ou ces centres commerciaux. Les propriétaires de ces usines situées en centre-ville sont contents qu'un bâtiment abandonné leur rapporte de l'argent en les vendant aux développeurs privés.

De plus, en Inde, toute loi relative au territoire (la division du terrain, l'aménagement du territoire, etc.) est établie par le gouvernement local, c'est-àdire que les législations de l'aménagement du territoire ne sont pas homogènes dans le pays. Un des phénomènes marquants d'aménagement du territoire tel qu'il est pratiqué en Inde est la préférence à développer de "nouveaux" quartiers au lieu de développer autour des "anciens" quartiers. Ainsi, ces structures sont souvent complètement détruites et le terrain est réutilisé. Un besoin urgent d'actualiser ces lois se présente donc. Les forges de Kumaon et Burwah ne sont pas "protégées" et si l'État d'Uttar 
Pradesh ou de Madhya Pradesh décident de développer le terrain, ils ont le droit de le faire et un patrimoine industriel exemplaire du transfert de technologie sera rasé de l'histoire du pays.

Dans cette époque de développement économique et d'urbanisation rapide que l'Inde vit actuellement, le patrimoine en général est soumis au risque d'être effacé sans laisser de trace et avant même d'avoir été identifié en ce qui concerne le patrimoine industriel. Il faut donc reconnaître que son inventaire et sa mise en valeur reste indispensable à la compréhension du développement industriel et technique de l'Inde.

${ }^{1}$ BEGLEY Vimala et DE PUMA Richard Daniel, Rome and India: The Ancient Sea Trade, Madison, Wisconsin, The University of Wisconsin Press, 1991.

${ }^{2}$ The Periplus of the Erythraean Sea, Travel and Trade in the Indian Ocean by a Merchant of the First Century. Trad. Wilfred H.Schoff, Longmans Green \& Co, New York, 1912.

3 BEGLEY et DE PUMA, 1991, op.cit.

4 DAUMAS Maurice, Histoire générale des techniques, Tome 1, Presses universitaires de France, 1962.

5 EDELBLUTTE Simon, «Paysages et territoires du patrimoine industriel au Royaume-Uni ", Revue Géographique del'Est [En ligne], vol. 48 / 1-2 | 2008, http://rge.revues.org/1165, consulté le 05 mai 2013.

${ }^{6}$ DOREL-FERRE Garcia, « Education et patrimoine : problématiques et pratiques du patrimoine industriel », Bulletin de Liaison des Professeurs d'Histoire Géographie de l'Académie de Reims, $\mathrm{n}^{\circ}$ 29-30, mars 2003, pg 23-26.

7 TICCIH, Charte Nizhny Tagil pour Le Patrimoine Industriel / Juillet, 2003.

8 TIWARI Rakesh, "The origins of Iron-working in India: New evidence from the Central Ganga Plain and the Eastern Vindhyas", http://www.archaeologyonline.net/artifacts/ironore.html, consulté le 25 avril 2013.

9 Cette expression a été inventée par Benjamin Disraeli, le premier ministre britannique. Au cours de son mandat - entre 1874 et 1881 - ses politiques ont incité l'expansion de l'empire britannique. C'est lors de cette conquête du monde que Disraeli a appelé l'Inde the "brightest jewel in the crown" ou le «joyaux le plus brillant de la couronne». De 1876 jusqu'au 1947, l'Inde était gouvernée par la monarchie britannique.

${ }^{10} \mathrm{http}$ ///theory.tifr.res.in/bombay/history/cotton.html, consulté le 25 avril 2013.
${ }^{11}$ http://theory.tifr.res.in/bombay/history/cotton.html, consulté le 25 avril 2013.

12 http://www.spiegel.de/international/spiegel/the-paradox-ofmumbai-slums-stocks-stars-and-the-new-india-a-4690312.html, consulté le 25 avril 2013.

${ }_{13}$ http://www.rediff.com/news/2007/jan/18sld3.htm, consulté le 25 avril 2013.

14 http://www.thehindubusinessline.com/todays-paper/tpcorporate/phoenix-mills-setting-up-luxury-mall-inmumbai/article1624402.ece?ref=archive, consulté le 25 avril 2013.

${ }^{15}$ AF GEIJERSTAM Jan, Landscapes of Technology Transfer: Swedish Ironmakers in India 1860-1864, Stockholm, Jemkontoret, 2004.

16 http://whc.unesco.org/fr/list/944/, consulté le 25 février 2013.

${ }^{17}$ C'est ainsi que décrit l'UNESCO la construction de ce chemin de fer ainsi dans la liste du patrimoine mondial. http://whc.unesco.org/fr/list/944/, consulté le 25 février 2013.

$18 \mathrm{http}: / /$ whc.unesco.org/uploads/nominations/944ter.pdf, consulté le 25 avril 2013.

${ }^{19}$ La compagnie Enfield débute en 1880 dans le domaine de la construction de bicyclettes. Un contrat gouvernemental pour fabriquer d'armes légères lui autorise à ajouter le «Royal » devant le nom de la société ainsi devenant Royal Enfield.

20 "Bhopal trial: Eight convicted over India gas disaster", BBC News, 7 June 2010.

${ }^{21}$ http://whc.unesco.org/en/list/, consulté le 25 avril 2013. 


\section{Bibliographie}

The Periplus of the Erythraean Sea, Travel and Trade in the Indian Ocean by a Merchant of the First Century. Trad. Wilfred H. Schoff, Longmans Green \& Co, New York, 1912.

AF GEIJERSTAM Jan, Landscapes of Technology Transfer: Swedish Ironmakers in India 1860-1864, Stockholm, Jemkontoret, 2004.

BEGLEY Vimala et DE PUMA Richard Daniel, Rome and India : The Ancient Sea Trade, Madison, Wisconsin, The University of Wisconsin Press, 1991.

BIRDWOOD George C. M, Industrial Arts of India, Londres, Chapman and Hall, 1980.

DAUMAS Maurice, Histoire générale des techniques, Tome 1, Presses universitaires de France, 1962.

DOREL-FERRE Garcia, «Education et patrimoine: problématiques et pratiques du patrimoine industriel », Bulletin de Liaison des Professeurs d'Histoire Géographie de l'Académie de Reims, ${ }^{\circ} 29-30$, mars 2003, pg 23-26.

EDELBLUTTE Simon, « Paysages et territoires du patrimoine industriel au Royaume-Uni », Revue Géographique de l'Est [En ligne], vol. 48 / 1-2 | 2008, http://rge.revues.org/1165, consulté le 05 mai 2013.

MAHIAS Marie-Claude, Le barattage du monde. Essais d'anthropologie des techniques en Inde. Paris, Fondation Maison des Sciences de l'Homme, 2002.

FILLIOZAT Jean, La Doctrine classique de la médecine indienne. Ses origines et ses parallèles grecs, Paris, Imprimerie Nationale, 1949 (2e édition, Paris, Ecole Française d'Extrême-Orient, 1975).

TIWARI Rakesh, "The origins of Iron-working in India: New evidence from the Central Ganga Plain and the Eastern Vindhyas", http://www.archaeologyonline.net/artifacts/ironore.html, consulté le 25 avril 2013

\section{Webographie}

http://theory.tifr.res.in/bombay/history/cotton.ht $\underline{\mathrm{ml}}$, consulté le 25 avril 2013.

http://www.spiegel.de/international/spiegel/theparadox-of-mumbai-slums-stocks-stars-and-thenew-india-a-469031-2.html, consulté le 25 avril 2013.

http://www.rediff.com/news/2007/jan/18sld3.htm , consulté le 25 avril 2013.

http://www.thehindubusinessline.com/todayspaper/tp-corporate/phoenix-mills-setting-upluxury-mall-inmumbai/article1624402.ece?ref=archive, consulté le 25 avril 2013.

http://www.whc.unesco.org, consulté le 25 avril 2013.

http://www.bmhrc.org/Bhopal\%20Gas\%20Tragedy .htm, consulté le 10 octobre 2013. 